\title{
Influence des systèmes de culture sur les risques d'érosion par ruissellement concentré. I. - Ana- lyse des conditions de déclenchement de l'érosion
}

\author{
Jean BOIFFIN, François PAPY (*), Micheline EIMBERCK $\left({ }^{* *}\right)$ \\ INRA, Station d'Agronomie de Laon-Péronne, rue Fernand Christ, BP 101,02004 Laon Cedex \\ (*) INRA, Unité de Recherches sur les Systèmes Agraires at le Développement. Centre de Recherches de \\ Grignon-Massv-Paris, 78850 Thiverval-Grignon \\ (**) INRA, Département de Science du sol, route de Saint-Cyr, 78000 Versailles
}

RÉSUMÉ

On a cherché à mettre en évidence les conditions d'apparition des rigoles et ravines temporaires typiques de l'érosion par ruissellement concentré qui prévaut dans les régions limoneuses du Nord-Ouest de l'Europe. Pour cela, on a observé pendant 3 campagnes, le comportement de 9 petits bassins-versants du Pays de Caux. L'apparition et l'aggravation des figures d'érosion sont mises en relation avec les caractéristiques des pluies et avec les états de surface des parcelles constituant l'impluvium. Il apparaît que la probabilité dérosion dépend étroitement du degré de développement des croûtes de battance, de la rugosité de surface, et de la présence d'empreintes de roues à la surface du sol. Lorsque l'érosion est provoquée par le ruissellement concentré, c'est donc sur la base de ces critères qu'il faut apprécier l'influence des systèmes de culture. En effet, ceux-ci induisent une forte variabilité spatiale et chronologique des propriétés hydrauliques du terrain.

Mots clés additionnels : Battance, bassin-versant, infiltrabilité, rugosité, détention superficielle, rigole, ravine temporaire, traces de roues. for initiating erosion.

In the context of the intensive agriculture of North-West Europe, runoff generation and concentration are fundamental processes that must be taken into account in order to understand and predict soil erosion. For assessing the impact of cropping systems (defined as plant sequences plus cropping management applied to each crop), it is necessary to consider their influence on the hydrodynamic properties and the microtopography of the soil surface. This is not explicitly done in the U.S.L.E. standard procedures. which in contrast put a major - and perhaps exaggerated stress on the effect of soil covering by the crop canopy. For a catchment area composed of several cultivated plots, the cropping systems applied to each one induce different types of spatial and temporal variation affecting infiltrability and surface storage. Some of these systematic variations are progressive, others discontinuous. Furthermore, the cropping system contributes to the creation of a specific network of channels for overland flow, this network also evolving with time. In order to reduce this complexity, a qualitative typology of soil surface states is proposed. 9 elementary watersheds, ranging from 0.6 to 13 ha, with gentle slopes and loamy soils, were observed in the Pays de Caux area during 3 cropping seasons. Gully initiation and development was not related with rainfall characteristics, but was clearly explained by the soil surface state of the agricultural plots constituting the catchment. This could lead us to an improvement of the system used for characterizing and grading cropping systems according to their influence on erosion.

Additional key words : Concentrated runoff. soil crusting, watershed. infiltrability, surface roughness, surface storage, rill, ephemeral gully, wheeltracks. 


\section{INTRODUCTION}

Dans les régions de plateaux limoneux du Nord-Ouest de l'Europe, et en particulier au Nord du Bassin Parisien, l'érosion des terres agricoles a pour principale origine le processus de concentration du ruissellement (BOIFFIn et al., 1986; MONNIER et al., 1986; AUZET, 1987). Celui-ci donne lieu à la formation de rigoles ou ravines temporaires largement espacées, et localisées de façon systématique dans des emplacements prédéterminés par la topographie. De telles formes d'érosion sont redevables d'une analyse spécifique (FOSTER et al., 1981, 1985 ; SPOMER \& HJelmFelt, 1986) différente de celle qui sous-tend l'équation universelle des pertes en terre, dite U.S.L.E. (WISCHMEIER \& SMITH, 1965).

En effet, celle-ci s'applique à des portions de versants indépendantes les unes des autres, alors que l'érosion par ruissellement concentré ne peut être appréhendée qu'au niveau plus global du bassin versant élémentaire. D'autre part, la hiérarchie des mécanismes qui interviennent dans le détachement de matière solide est différente: dans le cas de l'érosion par ruissellement concentré, celui-ci a pour origine la force tractrice exercée sur le sol par les écoulements; alors que dans le cas de l'érosion de versant, l'impact des gouttes de pluie intervient pour une part importante. Dans certains cas limites, par exemple avec de faibles longueurs de pente, il peut même être l'agent principal, voire exclusif, du détachement (Ellison, 1954; Young \& Wiersma, 1973; Hudson, 1981).

Il en résulte que la manière d'apprécier l'influence des systèmes de culture sur les risques d'érosion doit elle aussi être réexaminée en fonction du contexte. Ainsi. pour les situations à ruissellement concentré, l'effet protecteur du couvert végétal n'est peut être pas aussi important et universel qu'on l'admet généralement. Inversement, il convient sans doute d'accorder plus d'attention aux caractéristiques du terrain qui conditionnent les débits de ruissellement et leurs variations dans le temps (MONNIER \& BOIFFIn, 1986; MONNIER et al., 1986) : infiltrabilité, détention superficielle, existence et densité d'un réseau de collecte permettant un regroupement plus ou moins rapide des écoulements, sans oublier la résistance du sol à l'arrachement.

Ces propriétés sont sous la dépendance des caractéristiques hydriques et structurales des couches superficielles du sol. Vis-à-vis de l'infiltrabilité, ce sont même les tout premiers millimètres à partir de la surface qui jouent le rôle prépondérant (MONNIER et al., 1986).

Or, la mise en œuvre d'un système de culture sur une parcelle se traduit par la répétition plus ou moins régulière d'opérations culturales spécifiques, et de phases de croissance des cultures, qui ont, dans tous les cas, une action plus ou moins marquée sur l'état physique du sol. Le système de culture induit donc une sorte de cycle d'états de surface, qui comporte à certains moments de véritables discontinuités dans l'évolution des propriétés physiques (par exemple, brutal accroissement de l'infiltrabilité après un travail du sol), et, à d'autres, des évolutions plus progressives (par exemple, en sens inverse, décroissance de l'infiltrabilité par formation de croûtes superficielles). Dans l'espace, le système de culture induit également des hétérogénéités systématiques dont certaines (par exemple, les empreintes de roues) sont susceptibles de jouer un grand rôle vis-à-vis de la formation et la circulation du ruissellement (VOORHEEs et al., 1979; FULLEN, 1985).

Il en résulte une grande complexité qui, en l'état actuel des méthodes et des moyens de mesures physiques, rend difficile une approche totalement déterministe du rôle des systèmes de culture. Cependant, l'expérience montre qu'il existe, dans une région et à un moment donné, un nombre limité de grands types d'états de surface liés aux cultures présentes dans cette région et à la nature des opérations culturales qui leur sont appliquées (BOIFFIN et al., 1986).

L'objet du travail qui suit est de montrer que la prise en compte, même qualitative, de ces grands types d'état de surface et de leurs principales caractéristiques contribue à la compréhension de l'érosion par ruissellement concentré. Cette démarche fournira la base d'une analyse du rôle des systèmes de culture, mieux adaptée que l'approche classique, dérivée de l'U.S.L.E., au contexte des régions d'agriculture intensive du NordOuest de l'Europe.

\section{MATÉRIEL ET MÉTHODES}

\section{A. Principe de la démarche}

Il consiste à tenter de mettre en relation la chronologie d'apparition et de développement des symptômes d'érosion avec un suivi des pluies et des états de surface des terrains cultivés.

1. L'unité spatiale de base, on l'appellera bassin versant élémentaire, elle correspond aux ramifications ultimes du réseau hydrographique temporaire qui peut se créer lors des pluies. Elle est délimitée par un périmètre isolé hydrauliquement et par un exutoire unique plus ou moins large mais bien défini. Dans le contexte géomorphologique et agricole où l'on se situe, l'aire des « bassins versants élémentaires " ainsi identifiés est de l'ordre de 1 hectare à quelques dizaines d'hectares. En leur sein, on délimite et caractérise de manière distincte 3 domaines fonctionnels :

la plus grande partie de l'impluvium est à considérer essentiellement du point de vue de la genèse de l'excès d'eau superficiel qui, s'il se forme, peut être en totalité ou en partie stocké à la surface. Le surplus éventuel s'écoule sous forme d'un ruissellement qui reste diffus et non érosif par lui-même tant que les pentes sont inférieures à une certaine limite (4-5 p. 100 dans le cas des sols limoneux, d'après Savat \& De Ploey, 1982 ; Govers, 1985) ;

en contrebas se situent un ou quelques collecteurs principaux qui sont des talwegs ou des fourrières. C'est au niveau de ces zones de concentration finale que se situe l'essentiel des pertes en terre;

- entre ces 2 domaines, le ruissellement est acheminé par un réseau de collecte, souvent constitué par les empreintes de roues. Selon les conditions, ce réseau de collecte est ou non lui-même affecté par des incisions, en général plus marquées à proximité du collecteur principal. 
Un quatrième domaine, important à considérer du point de vue des nuisances, est la zone d'atterrissement. Selon les cas, elle peut se situer en partie ou en totalité à l'extérieur du bassin versant et à des distances plus ou moins grandes. Nous avons donc renoncé à intégrer son observation dans le suivi de l'érosion.

Le périmètre d'isolement hydraulique peut se situer au sein d'une seule parcelle agricole ou, au contraire, recouper les limites de plusieurs parcelles. Lorsque la zone d'émission du ruissellement et le collecteur principal qu'elle alimente sont intégralement situés dans la même parcelle agricole, on parlera de bassin versant élémentaire simple. Dans le cas contraire, c'est-à-dire lorsqu'il y a communication entre plusieurs parcelles, on parlera de bassin versant élémentaire composite. Lorsque les bassins versants recoupent plusieurs parcelles, il se peut qu'à certaines périodes de l'année ces parcelles ne communiquent pas entre elles; on a alors une juxtaposition de bassins versants élémentaires simples. Une fois toutes les communications établies, le bassin versant fonctionne en tant qu'unité composite.

Il est donc essentiel, vis-à-vis de l'objectif annoncé, d'identifier quelles sont, au sein de l'impluvium potentiel, les zones d'émission du ruissellement qui alimentent effectivement les collecteurs principaux. Ce diagnostic peut être fait en observant le tracé des figures d'érosion et en vérifiant leur continuité au niveau des limites entre parcelles. Il exige un bon suivi des opérations culturales car certaines interventions peuvent effacer un tronçon de rigole: elles font alors disparaître une continuité dont il faut tenir compte pour interpréter correctement la genèse de cette figure d'érosion ; c'est-à-dire la mettre en correspondance avec l'impluvium ayant effectivement émis le ruissellement qui l'a créée.

2. Sur le plan chronologique, le point de départ des observations correspond à une date à laquelle l'état physique du sol est bouleversé, sur une partie importante du bassin versant, par un ensemble d'opérations culturales ayant également pour effet d'effacer les figures d'érosion préexistantes. Il s'agit d'opérations de travail du sol, de semis ou de récolte (arrachages avec roulages).

Le suivi est interrompu lorsque les moyens d'observation rapides utilisés sur le terrain ne permettent plus de détecter une évolution sensible des figures d'érosion. Ainsi, l'analyse qui suit porte essentiellement sur le déclenchement et les premières étapes d'aggravation de l'érosion.

Entre le début et la fin du suivi, l'entité chronologique de base sur laquelle portent les observations est en principe «la pluie », définie comme une séquence ininterrompue de précipitations. La pluie est découpée en épisodes d'intensité homogène mais variable entre 2 épisodes consécutifs. Avant la pluie, est effectuée l'observation des états de surface des parcelles constituant le bassin versant élémentaire. Après la pluie, on note les symptômes d'érosion. Dans certains cas, si les observateurs n'ont pas eu la possibilité d'intervenir entre 2 pluies, les observations doivent en fait être rapportées à un groupe de pluies.

L'interprétation de l'effet des précipitations est évidemment d'autant moins précise que le groupe de pluies considéré est plus important et comporte plus d'interruptions.

\section{B. Choix et caractérisation des bassins versants élémen- taires}

L'étude a été réalisée dans le Pays de Caux, région située au Nord-Ouest du Bassin Parisien en bordure de la Manche, entre Le Havre et Dieppe. Deux communes ont été choisies en raison de la gravité des problèmes d'érosion: Fongueusemare et Epouville. Pour une description détaillée des caractéristiques du milieu, on se reportera à BoIfFin et al. (1986). On se bornera ici à rappeler que le Pays de Caux est un vaste plateau reposant sur la craie du Turonien et du Sénonien, recouvert d'une épaisseur importante de limons éoliens. Le relief général est mollement ondulé, avec de nombreux talwegs et vallées sèches peu inclinés sauf au voisinage de la mer et de la Seine. Les sols du plateau sont homogènes; toutes les parcelles étudiées ici présentent une texture limoneuse (teneurs en argile comprises entre 11,5 et 14 p. 100) dont résulte un comportement extrêmement battant. Les teneurs en matière organique ne sont en général pas suffisantes pour modifier cette tendance: 88 p. 100 des parcelles étudiées ont des teneurs inférieures à 2 p. 100 . La vitesse de formation des croûtes n'est réduite de façon perceptible qu'en cas de retournement très récent de prairies (BoIfFIN et al., 1986), correspondant à des teneurs supérieures à 2,5 p. 100. Par ailleurs, le ressuyage de la couche labourée est généralement rapide et aucun des sols observés ne présente de manifestation d'hydromorphie. 95 p. 100 des valeurs de $\mathrm{pH}$ sont inférieures à 7.

Une présentation sommaire des principales caractéristiques topographiques des 9 bassins versants élémentaires étudiés est donnée par le tableau 1 . On vérifie que les topographies sont dans l'ensemble peu inclinées, mais on note, par ailleurs, une forte variabilité des longueurs de pente et des aires d'impluvium. Les bassins versants $n^{\text {os }} 1,3$ et 5 sont constitués d'une seule parcelle agricole, alors que les autres sont constitués de plusieurs parcelles qui, selon les circonstances, communiquent ou non entre elles.

Ces bassins versants ont été suivis lors des 3 principales campagnes d'observation: printemps 1985 (P85), automne-hiver 1985-86 (A85), automne-hiver 1986-87 (A86). Des observations plus sommaires ont également été réalisées lors des printemps 1986 et 87.

Malgré la présence d'élevage dans certaines exploitations concernées, les systèmes de culture appliqués aux sites étudiés ne comportent que des plantes annuelles avec une forte proportion de cultures sarclées de printemps. Il en résulte que l'occupation du sol au sein des bassins versants élémentaires, peut être décrite en se ramenant à un nombre limité de cas-types (tabl. 2). Chacun d'eux admet un certain nombre de variantes, dont l'existence sur le terrain est d'ailleurs plus ou moins fugace. Par exemple, du fait de l'étalement des dates d'arrachage de betterave, les semis du blé suivant s'effectuent souvent en plusieurs fois, et un bassin versant correspondant a priori à une seule parcelle, doit alors être considéré temporairement comme constitué de plusieurs parcelles de caractéristiques très différentes.

\section{Enregistrement des pluies}

Il est réalisé, sur chaque commune, à l'aide d'un pluviographe à augets basculeurs et enregistrement 
TABLEAU 1

Principales caractéristiques topographiques des bassins versants étudiés.

Topographical characteristics of the observed watersheds.

\begin{tabular}{|c|c|c|c|c|c|c|c|c|c|c|c|c|}
\hline $\mathrm{N}^{0}$ du site & & 1 & 2 & 3 & & & & 5 & 6 & 7 & 8 & 9 \\
\hline $\begin{array}{l}\text { Type de collecteur } \\
\text { principal }\end{array}$ & Talweg & Fourrière & Talweg & Talweg & Talweg & Fourrière & \multicolumn{2}{|c|}{ Fourrière } & Talweg & Talweg & Talweg & Talweg \\
\hline $\begin{array}{l}\text { Surface drainée } \\
\text { (ha) }\end{array}$ & 2,8 & 4 & 10 & 13 & 9,7 & 0,6 & 1,5 & 3 & 11 & 10 & 5,6 & 7,5 à 8 \\
\hline $\begin{array}{l}\text { Pente du } \\
\text { collecteur }(\%)\end{array}$ & 6 & 5 & $2 \dot{a} 6$ & 2 à 6 & 3 à 5 & 2 à 5 & 4 à 5 & 5 & 1 à 5 & 4 à 7 & 4 & 2 à 4 \\
\hline $\begin{array}{l}\text { Pente } \\
\text { transversale }(\%)\end{array}$ & 1 à 3 & 1 & 1 à 3 & 1 à 2 & 1 à 3 & 1 à 2 & 1 à 2 & 2 & 1 à 2 & 1 à 2 & 1 & 1 \\
\hline $\begin{array}{l}\text { Longueur maximale } \\
\text { des écoulements }(\mathrm{m})\end{array}$ & 120 & 250 & 250 & 100 à 200 & 250 à 300 & $>300$ & 50 & 50 & 250 à 400 & 250 & 50 & 170 \\
\hline $\begin{array}{l}\text { Nombre de parcelles } \\
\text { agricoles de } \\
\text { l'impluvium }\end{array}$ & 1 & 1 & 4 & 1 & 2 & 1 & 1 & 1 & 4 & 3 & 2 & 3 \\
\hline
\end{tabular}

TABLEAU 2

Occupation du sol sur les bassins versants étudiés.

Land occupation on the observed watersheds.

\begin{tabular}{|c|c|c|c|c|}
\hline & \multicolumn{2}{|c|}{ Types de situations culturales } & Campagnes d'observations & Sites correspondants $\left({ }^{* *}\right)$ \\
\hline \multirow{12}{*}{$\begin{array}{l}\text { Bassins } \\
\text { versants } \\
\text { simples }\end{array}$} & \multirow{3}{*}{$\begin{array}{l}\text { Semis } \\
\text { d'automne }\end{array}$} & Blés & A85 & \\
\hline & & & A86 & $2 \mathrm{D}, 4,8,6$ \\
\hline & & $\begin{array}{l}\text { Colza } \\
\text { Escourgeon }\end{array}$ & $\begin{array}{l}\text { A } 86 \\
\text { A } 86\end{array}$ & $\begin{array}{l}7 \\
7\end{array}$ \\
\hline & \multirow{4}{*}{$\begin{array}{l}\text { Interculture } \\
\text { céréales } \\
- \\
\text { culture de } \\
\text { printemps }\end{array}$} & Déchaumages à dents & $\begin{array}{l}\text { A } 85 \\
\text { A } 86\end{array}$ & $\begin{array}{l}4 \\
3,6\end{array}$ \\
\hline & & Déchaumages à socs & A 86 & 3,6 \\
\hline & & Chaumes non travaillés & A86 & 3,6 \\
\hline & & Engrais verts & A 86 & 6 \\
\hline & \multirow{3}{*}{$\begin{array}{l}\text { Culture } \\
\text { de } \\
\text { printemps }\end{array}$} & Pois & P87 & 9 \\
\hline & & $\begin{array}{l}\text { Betterave sucrière } \\
\text { (avant binage) } \\
\text { (après binage) }\end{array}$ & $\begin{array}{l}\text { P85 } \\
\text { P86 }\end{array}$ & $\begin{array}{l}1,2,6,7 \\
4\end{array}$ \\
\hline & & Pomme de terre & P85 & 3,6 \\
\hline & \multirow{2}{*}{$\begin{array}{l}\text { Interculture } \\
\text { culture de } \\
\text { printemps - } \\
\text { culture de } \\
\text { printemps }\end{array}$} & Après « déchaumage» sur lin & A 85 & 5 \\
\hline & & $\begin{array}{l}\text { Après récolte de } \\
\text { pomme de terre }\end{array}$ & A 85 & 6 \\
\hline \multirow{4}{*}{$\begin{array}{l}\text { Bassins (*) } \\
\text { versants } \\
\text { composites }\end{array}$} & \multicolumn{2}{|c|}{$\begin{array}{l}\text { Interculture après récolte de betterave } \\
\text { sucrière ou pomme de terre/semis de blé }\end{array}$} & $\begin{array}{l}\text { A85 } \\
\text { A86 }\end{array}$ & $\begin{array}{l}6,7 \\
2 \mathrm{EF}, 4\end{array}$ \\
\hline & \multicolumn{2}{|c|}{$\begin{array}{l}\text { Sol travaillé après récolte d'automne } \\
\text { /déchaumage de blé }\end{array}$} & A 85 & 1,2 \\
\hline & \multicolumn{2}{|c|}{ Semis de blé/semis de blé plus précoce } & A85 & 7,1 \\
\hline & \multicolumn{2}{|c|}{ Semis de blé/semis de blé plus tardif } & non observé & \\
\hline
\end{tabular}

$\left({ }^{*}\right)$ Parcelle amont/parcelle aval.

(**) Les numéros renvoient aux bassins versants décrits dans le tableau 1. Les lettres majuscules désignent des subdivisions si les bassins versants contiennent plusieurs parcelles. 
TABLEAU 3

Variation des caractéristiques des pluies observées.

Variability of the observed rainfall characteristics.

\begin{tabular}{|c|c|c|c|c|c|c|c|c|}
\hline \multirow{2}{*}{$\begin{array}{l}\text { Campagnes } \\
\text { d'observations }\end{array}$} & \multicolumn{2}{|c|}{$\begin{array}{l}\text { Hauteurs totales } \\
\text { cn mm }\end{array}$} & \multicolumn{2}{|c|}{$\begin{array}{l}\text { Hauteurs à intensité } \\
>5 \mathrm{~mm} / \mathrm{h}(\mathrm{mm})\end{array}$} & \multicolumn{2}{|c|}{$\begin{array}{l}\text { Hauteurs à intensité } \\
>10 \mathrm{~mm} / \mathrm{h}(\mathrm{mm})\end{array}$} & \multicolumn{2}{|c|}{$\begin{array}{c}\text { Intensités de } \\
\text { pointe }(\mathrm{mm} / \mathrm{h})\left({ }^{*}\right)\end{array}$} \\
\hline & Mini & Maxi & Mini & Maxi & Mini & Maxi & Mini & Maxi \\
\hline Printemps 85 & 6 & 19 & 0 & 11 & 0 & 7 & 1,5 & 36 \\
\hline Automne 85 & 4,2 & 30,5 & 1,0 & 13,5 & 0 & $1 i, 1$ & 6 & 31 \\
\hline Automne 86 & $\leqslant 6$ & 19.8 & 0 & 13,3 & 0 & 8,6 & 3,3 & 72 \\
\hline
\end{tabular}

(*) Pour au moins $1 \mathrm{~mm}$.

mécanique dont la résolution est de $0,1 \mathrm{~mm}$ pour les hauteurs de pluies, $1 \mathrm{mn}$ environ pour le temps. On accède donc aux intensités de chaque épisode avec une précision acceptable, tout au moins lorsque les hauteurs d'eau considérées pour chaque épisode sont supérieures ou égales au millimètre et les intensités inférieures à environ $30 \mathrm{~mm} / \mathrm{h}$.

Le tableau 3 montre la grande variabilité des caractéristiques des pluies ou groupes de pluies correspondant à des observations sur le terrain. Cependant, cet échantillon ne contient pas d'événement de type exceptionnel, les plus fortes pluies enregistrées se caractérisant par des couples intensité-durée de fréquence au moins quinquennale.

\section{Description des états de surface}

Le but de cette description n'est pas de fournir, dans l'absolu, une mesure des propriétés physiques contrôlant la formation et l'érosivité du ruissellement, mais de détecter les évolutions et les différences entre parcelles. De plus, il est souhaitable d'observer très rapidement après une pluie un maximum de parcelles. On a donc eu recours à un système de notations aussi synthétique que possible. Quatre catégories de critères font l'objet de notations, dont les modalités sont de type qualitatif ordonné.

\section{Critères relatifs à l'infiltrabilité}

Une fois le sol humecté, celle-ci dépend principalement :

- du stade de développement des croûtes de battance (BOIFFIN, 1984, 1986; BOIFFIn \& MONNIER, 1986). Une notation simple et fiable consiste à identifier 5 faciès typiques (Bolfrin, 1984 ; BolFrIN \& BRESSON, 1987) obtenus au cours de cette formation (tabl. 4);

- de la présence d'empreintes de roues non effacées, induisant une porosité structurale très faible dès la surface du sol. Ces empreintes correspondent à des traitements phytosanitaires, à des épandages de matières fertilisantes, à des opérations de semis ou de récolte. En mesurant les largeurs et les espacements des empreintes, on calcule le pourcentage de la surface du sol affectée.

\section{Critères relatifs à la détention superficielle}

Rappelons qu'il s'agit de la capacité de stockage temporaire à la surface du sol. Elle a une forte influence
TABLEAU 4

Notation du stade de développement des croûtes de battance. Scoring of soil crusting stages.

\begin{tabular}{llc}
\hline Code & Faciès morphologique & $\begin{array}{c}\text { Gamme de variation probable } \\
\text { de l'infiltrabilité }(\mathrm{mm} / \mathrm{h}) \\
\text { (sol limoneux et humide) }\end{array}$ \\
\hline F0 & $\begin{array}{l}\text { Faciès fragmentaire } \\
\text { initial }\end{array}$ & $30-50$ \\
\hline F1 & $\begin{array}{l}\text { Faciès fragmentaire } \\
\text { altérè à } \\
\text { croûtes structurales }\end{array}$ & $5-30$ \\
\hline F1-F2 & $\begin{array}{l}\text { Faciès de transition } \\
\text { (apparition } \\
\text { locale de croûtes de } \\
\text { dépôt) }\end{array}$ \\
\hline F2 & $\begin{array}{l}\text { Faciès continu à } \\
\text { croûtes de dépôt }\end{array}$ \\
\hline F3 & $\begin{array}{l}\text { Faciès à croûtcs rema- } \\
\text { niées (agents } \\
\text { climatiques et biologi- } \\
\text { ques) }\end{array}$ \\
\hline \hline
\end{tabular}

sur la hauteur d'eau qui ruisselle, non seulement par soustraction directe du volume stocké, mais aussi par son effet indirect sur la capacité d'infiltration, accrue par l'élévation de la pression hydrostatique au niveau des flaques (Monteith, 1974; Mitchell \& Jones, 1978 ; Cogo et al., 1984 ; Steichen, 1984). Plusieurs auteurs ont tenté de modéliser cette capacité de stockage et de la relier à l'évaluation la plus courante de la rugosité, c'est-à-dire l'écart-type des cotes de surfaces par rapport à un niveau de référence. En comparant différentes techniques culturales, MOORE \& LARSON (1979) obtiennent une gamme de rugosité qui va de 0,6 à $2,6 \mathrm{~cm}$ et évaluent des capacités de stockage allant de 0,2 à $21 \mathrm{~mm}$. ONSTAD (1984), prenant en considération la pente et des hypothèses de modélisation différentes, calcule des valeurs 2 à 3 fois plus faibles pour une rugosité identique et une pente de 2 p. 100.

De nombreux auteurs (par exemple CoGo et al., 1984 ; ZOBECK \& ONSTAD, 1987) ont évalué l'influence des techniques de travail du sol sur la rugosité. Après travail du sol, l'action dégradante des pluies engendre une diminution progressive de celle-ci (BOIFFIN \& SEBILLOTTE, 1976 ; DeXTER, 1977 ; BOIFFIN, 1984). Dans le cadre d'observations au champ, on a distingué 4 classes 
(tabl. 5), cette évaluation étant faite compte tenu du sens probable des écoulements. On s'est assuré à la fois de la reproductibilité de cette notation entre différents observateurs et de sa bonne correspondance avec les mesures effectuées par aspérimétrie.

TABLEAU 5

Signification des notations de rugosité utilisées.

Meaning of the surface roughness grades.

\begin{tabular}{cccc}
\hline \hline $\begin{array}{c}\text { Classe } \\
\text { de rugosité }\end{array}$ & $\begin{array}{c}\text { Ecart-type } \\
\text { des cotes } \\
\text { de surface } \\
(\mathrm{cm})(1)\end{array}$ & $\begin{array}{c}\text { Détention } \\
\text { superficielle } \\
(\mathrm{mm})(2)\end{array}$ & $\begin{array}{c}\text { Exemple typique } \\
\text { de situation } \\
\text { correspondante }\end{array}$ \\
\hline 1 & $0-1,2$ & $0-1,5$ & $\begin{array}{l}\text { Semis très battus ou chanticrs } \\
\text { de récolte très compactés. } \\
\text { Semis légèrement battus. }\end{array}$ \\
2 & $1,2-2,0$ & $1,5-3$ & $\begin{array}{l}\text { Semis récents à structure mot- } \\
\text { teuse intacte ; sols travaillćs } \\
\text { sans résidus. } \\
\text { Labours récents ; déchauma- } \\
\text { ges récents à résidus abon- } \\
\text { dants }\end{array}$ \\
\hline
\end{tabular}

(1) Déterminé à l'aide d'un aspérimètre à aiguilles (Bolfrin, 1984). La rugosité est évaluée dans le sens de la dernière opération de tràvail du sol, supposé être celui des écoulements.

(2) Evaluation approximative en appliquant le modèle de ONSTAD (1984) pour une pente de $2 \%$.

\section{Critères relatifs à la circulation et au regroupement des écoulements}

Plus ces 2 processus sont réalisés rapidement, et plus vite sont atteints les débits de pointe au niveau des collecteurs principaux; plus élevé aussi est le risque d'incision dans les collecteurs adjacents. Le critère qui nous est apparu déterminant est la présence ou l'absence, à la surface du sol, de motifs linéaires continus et en dépression, disposés de manière à recouper les collecteurs principaux. Il s'agit soit d'empreintes de roues ron effacées, soit des sillons périodiques formés par certaines opérations de travail du sol (l'exemple typique est le buttage des pommes de terre). Compte tenu des objectifs mentionnés précédemment, le critère fruste de présence-absence a paru suffisant, dans un premier temps, d'autant que les espacements ne sont jamais très grands (maximum $24 \mathrm{~m}$ suite à des traitements phytosanitaires).

\section{Critères relatifs à la sensibilité du sol au détachemen par le ruissellement}

La plupart des références disponibles concernent des lits de sédiments non agrégés et mettent en évidence l'importance de la taille des particules. Dans le cas de sols cultivés, la cohésion joue un rôle prédominant (Savat, 1979; Rauws, 1987). Pour une texture et une humidité données, le principal facteur de variation de la détachabilité à considérer est donc la compacité (LyLE \& SMERdon, 1965). A défaut de mesures, trop longues à réaliser, on s'est contenté de distinguer les situations où la surface occupée par les traces de roues était respectivement inférieure ou supérieure à 50 p. 100 ; dans le premier cas, la couche arable est présumée moins compacte et résistante que dans le deuxième qui concerne essentiellement les chantiers de récolte non retravaillés.

\section{E. Classement des symptômes d'érosion}

Dans le même esprit que pour la description des états de surface, les observations de terrain consistent à relever, sur un plan, un schéma de localisation de ces symptômes, assorti de quelques mesures de profondeur et de largeur des rigoles. Pour chaque séance d'observation réalisée sur un bassin-versant déterminé, une appréciation globale à l'échelle de ce bassin versant conduit à attribuer aux symptômes d'érosion une note synthétique de gravité. Les 5 modalités retenues sont présentées en légende du tableau 7.

\section{RÉSULTATS ET DISCUSSION}

\section{A. Chronologie d'apparition des rigoles}

Elle est récapitulée, pour les 3 principales campagnes d'observation, dans le tableau 6. Il apparaît que, au sein de la même commune et pour une campagne donnée, l'érosion se déclenche, selon les sites, lors de 3 ou 4 dates privilégiées correspondant à des événements pluvieux marquants. Cependant, d'un site à l'autre au sein de la même commune (et donc avec sensiblement les mêmes pluies), les dates d'apparition des rigoles varient d'environ 2 mois; dans certains cas (intercultures faisant suite à une céréale), aucun symptôme d'érosion n'apparaît pendant plus de 8 mois. Cette variabilité semble en partie liée à la situation chronologique relative des pluies et des opérations culturales : ainsi, les semis les plus précoces du printemps 85 ont subi des pluies du 10 au 13 avril que n'ont pas reçues les semis postérieurs, ce qui peut expliquer le décalage observé dans l'apparition des rigoles. Mais des cas inverses peuvent être mentionnés, comme celui du site 7A semé en escourgeon le 30/09/86 et qui ne s'érode pas contrairement à des semis de blé plus tardifs. De plus, certaines surfaces travaillées presque au même moment (sites $5 \mathrm{~A}$ et $5 \mathrm{~B}$ à l'automne 85 ) se sont érodées à des dates très différentes.

Il y a donc lieu de procéder à une analyse plus détaillée des conditions de formation des rigoles, à la lumière des informations recueillies sur les principaux déterminants du ruissellement: les pluies et l'état de surface du terrain.

\section{B. Influence des caractéristiques des pluies}

Cette influence ne peut être mise en évidence que si les observations d'érosion ont pu avoir lieu juste avant et juste après une pluie bien déterminée. 14 événements pluvieux répondant strictement à ce critère ont pu être sélectionnés. Leurs caractéristiques et leurs conséquences sur l'érosion sont décrites dans le tableau 7. On note : 
TABLEAU 6

Dates d'apparition des symptômes d'érosion.

Dates of erosion symptom appearance.

\begin{tabular}{|c|c|c|c|c|}
\hline $\begin{array}{l}\text { Campagne } \\
\text { d'observation }\end{array}$ & Lieu & $\begin{array}{c}\text { Sites } \\
\text { observés }(*)\end{array}$ & $\begin{array}{l}\text { Opérations réalisées pendant } \\
\text { la campagne d'observation }\end{array}$ & $\begin{array}{l}\text { Dates de formation } \\
\text { des rigoles }\end{array}$ \\
\hline \multirow{7}{*}{ Printemps 1985} & Fongueusemare & 1 & Semis de betterave sucrière : $23 / 04$ & $20 / 05$ \\
\hline & & 2 & Semis de betterave sucrière : $20 / 04$ & $20 / 05$ \\
\hline & & 3 & Plantation de pomme de terre : $28 / 04$ & $20 / 05$ \\
\hline & Epouville & $6 \mathrm{~B}$ & Plantation de pomme de terre : $10 / 04$ & $13 / 04$ \\
\hline & & 1 & & \\
\hline & & $6 \mathrm{C}$ & Semis de betterave sucrière : $10 / 04$ & $13 / 04$ \\
\hline & & 7 & Semis de betterave sucrière: $17 / 04$ & cntre le 1 et $21 / 06$ \\
\hline \multirow{18}{*}{ Automne 1985} & Fongueusemare & $1 \mathrm{~A}$ & $\begin{array}{l}\text { b.s. en place puis récolte le } 3 / 11 \text {, } \\
\text { puis semis de blé successifs : du } 12 / 11\end{array}$ & $5 / 11$ \\
\hline & & 1 & à fin décembre & \\
\hline & & $1 \mathrm{~B}$ & $\begin{array}{l}\text { Déchaumage sur blé, paille enlcvée } \\
\text { (fin septembre) }\end{array}$ & $5 / 11$ \\
\hline & & $2 \mathrm{C}$ & $\begin{array}{l}\text { b.s. en place puis récoltée le } 18 / 11 \text {, } \\
\text { avec travail au chisel : } 18-28 / 11\end{array}$ & $5 / 01$ \\
\hline & & ! & & \\
\hline & & $2 \mathrm{DE}$ & $\begin{array}{l}\text { Déchaumage sur blé, paille enfouie: } \\
\text { le } 2 / 10\end{array}$ & $5 / 01$ \\
\hline & & 3 & Semis de blé : $15 / 10$ & $5 / 11$ \\
\hline & & 4 & $\begin{array}{l}\text { Déchaumage sur blé, paille enfouie : } 2 / 10 \\
\text { puis épandages de lisier (fin novembre) }\end{array}$ & $26-27 / 11$ \\
\hline & & $5 \mathrm{~A}$ & Déchaumage sur lin (outil à socs) : $7 / 10$ & $5 / 01$ \\
\hline & & $5 \mathrm{~B}$ & Déchaumage sur lin (outil à dents) : $2 / 10$ & $5 / 11$ \\
\hline & Epouville & $6 \mathrm{~B}$ & Chantier de récolte pdt laissé en l'état & $26-28 / 11$ \\
\hline & & 1 & & \\
\hline & & $6 \mathrm{C}$ & Semis de blé : le $8 / 11$ & $26-28 / 11$ \\
\hline & & 7Al & b.s. puis chantier de récolte de b.s. le & - \\
\hline & & 1 & $27 / 11$, puis semis de ble le $15 / 12$ & \\
\hline & & 7A2 & b.s. puis chantier de récolte de b.s. & $5 / 11$ \\
\hline & & 1 & début nov., puis semis de blé le $15 / 11$ & \\
\hline & & $7 \mathrm{~B}$ & Semis de blé le $18 / 10$ & $5 / 11$ \\
\hline \multirow{16}{*}{ Automne 1986} & Fongueusemare & $2 \mathrm{D}$ & Semis de blé : 6-7/10 & $11-15 / 12$ \\
\hline & & et & & \\
\hline & & $2 \mathrm{G}$ & b.s. récoltée vers le $15 / 11$ & - \\
\hline & & 1 & & \\
\hline & & $2 \mathrm{EF}$ & Semis de blé les $10-11 / 10$ & $18-28 / 11$ \\
\hline & & 3 & $\begin{array}{l}\text { Différentes modalités de déchaumage } \\
16 / 09 \text { et } 15 / 10\end{array}$ & $\begin{array}{l}\text { Aucune érosion } \\
\text { observéc }\end{array}$ \\
\hline & & $4 \mathrm{~A}$ & Récolte de b.s. du $22 / 10$ au début nov. & $20-25 / 11$ \\
\hline & & 1 & puis semis de blé le $8 / 11$ & \\
\hline & & $4 \mathrm{~B}$ & Semis de blé le $14 / 10$ & $19-20 / 10$ \\
\hline & & $4 \mathrm{C}$ & Scmis de blé le $14 / 10$ & $14-29 / 10$ \\
\hline & & 8 & Semis de blé le $10 / 10$ & $11-21 / 12$ \\
\hline & Epouville & $6 \mathrm{~A}$ & Semis de blé le $31: 10$ & $5-16 / 12$ \\
\hline & & $6 \mathrm{~B}$ & Semis de blé le $15 / 11$ & $17-30 / 12$ \\
\hline & & $6 \mathrm{C}$ & $\begin{array}{l}\text { Différentes modalités de déchaumage } \\
\text { et semis d'cngrais vert }\end{array}$ & $\begin{array}{l}\text { Aucune érosion } \\
\text { observéc }\end{array}$ \\
\hline & & $7 \mathrm{~A}$ & Semis d'escourgeon le $30 / 09$ & Aucune érosion obser. \\
\hline & & $7 \mathrm{~B}$ & Semis de colza le $12 / 09$ & $16-21 / 10$ \\
\hline Printemps 1987 & Fongueusemare & 9 & Semis de pois du 10 au $15 / 03$ & $18-30 / 03$ \\
\hline
\end{tabular}

(*) Mêmes codes que pour le tableau 2 : parcelles amont/parcelles aval. 
TABLEAU 7

Caractéristiques et conséquences sur l'érosion de quelques événements pluvieux. Characteristics of some rainfall events and their effects on erosion.

\begin{tabular}{|c|c|c|c|c|c|c|c|c|c|c|}
\hline \multirow[t]{2}{*}{ Dates } & \multirow[t]{2}{*}{ Commune (1) } & \multicolumn{3}{|c|}{ Hauteurs (mm) } & \multirow{2}{*}{$\begin{array}{c}\text { Intensité } \\
\text { maximale } \\
\text { pour au } \\
\text { moins } 1 \mathrm{~mm}\end{array}$} & \multicolumn{5}{|c|}{ Effets notés sur les sites ayant pu être observés (2) } \\
\hline & & Totale & avec & avec & & 0 & $\varepsilon$ & 1 & 2 & 3 \\
\hline \multicolumn{11}{|c|}{$I \geqslant 5 \mathrm{~mm} / \mathrm{h} \quad I \geqslant 10 \mathrm{~mm} / \mathrm{h}$} \\
\hline $20 / 05 / 85$ matin & $\mathrm{F}$ & 7,0 & 7,0 & 7,0 & 35 & $1,2 \mathrm{~A}, 3$ & & & \multirow{2}{*}{\multicolumn{2}{|c|}{$1,2 \mathrm{~A}, 3$}} \\
\hline $20 / 05 / 85$ soir & $\mathrm{F}$ & 6,0 & 6,0 & 6,0 & 36 & & & & & \\
\hline $13 / 04 / 85$ & E & 10,5 & 9,0 & 1,5 & 10 & & $6 \mathrm{~B}, 6 \mathrm{C}$ & & & \\
\hline $28 / 04 / 85$ & E & 8,5 & 0 & 0 & 1.5 & & & $6 \mathrm{~B}, 6 \mathrm{C}$ & & \\
\hline $12-13 / 05 / 85$ & $\mathrm{E}$ & 19,0 & 11,0 & 2,0 & 10 & 7 & & & $6 \mathrm{~B}, 6 \mathrm{C}$ & \\
\hline $5-6 / 11 / 85$ & $\mathrm{~F}$ & 21,7 & 13,5 & 11,1 & 31 & 2C. $2 \mathrm{DE}, 4,5 \mathrm{~A}$ & 3 & $1 \mathrm{~A}$ & $1 \mathrm{~B}, 5 \mathrm{~B}$ & \\
\hline $05 / 11 / 85$ & E & 12,0 & 9,5 & 8,4 & 25 & & & $7 \mathrm{~A}$ & $7 \mathrm{~B}$ & \\
\hline $11 / 11 / 85$ & E & 6,0 & 1,0 & 1,0 & 24 & $6 \mathrm{~B}, 6 \mathrm{C}$ & & & & \\
\hline $15 / 11 / 85$ & E & 4.2 & 1,8 & 0 & 6 & $7 \mathrm{~A} 1,7 \mathrm{~A} 2,7 \mathrm{~B}$ & & & & \\
\hline $26-27 / 11 / 85$ & $\mathrm{~F}$ & 19,3 & 7,7 & 2,4 & 11 & $\begin{array}{l}1 \mathrm{~A}, 1 \mathrm{~B}, 2 \mathrm{C} \\
2 \mathrm{DE}, 5 \mathrm{~A}\end{array}$ & 4 & $3,5 \mathrm{~B}$ & & \\
\hline $26-28 / 11 / 85$ & E & 22,0 & 9.6 & 2,4 & 12 & & $7 \mathrm{Al}$ & $7 \mathrm{~A} 2$ & $6 \mathrm{~B}$ & $6 \mathrm{C}$ \\
\hline $29-30 / 11 / 85$ & F & 10.5 & 2.2 & 0 & 9 & & & & & \\
\hline $05 / 01 / 86$ & $F$ & 30.5 & 12.0 & 0 & 6 & & $2 \mathrm{DE}, 4,5 \mathrm{~A}$ & $1 \mathrm{~B}, 2 \mathrm{C}$ & 1A, 3 & $5 B$ \\
\hline $05 / 01 / 86$ & E & 30,2 & 11,0 & 10,0 & 1 & $7 \mathrm{Al}$ & $7 \mathrm{~A} 2$ & $7 \mathrm{~B}$ & $6 \mathrm{~B}$ & $6 \mathrm{C}$ \\
\hline
\end{tabular}

(1) $\mathrm{F}=$ Fongueusemare $; \mathrm{E}=$ Epouville

(2) $\varepsilon=$ Griffes discontinues et peu profondes (quelques centimètres, pas d'exportation de terre à l'échelle décamétrique).

1 = Griffes et rigoles connectées et continues n'atteignant pas le fond du labour. Exportations à l"échelle hectométrique.

2 = Rigoles profondes (fond du labour), larges de 30 i $50 \mathrm{~cm}$.

3 = Rigoles entailant le fond du labour, atteignant à l'aval plus de $50 \mathrm{~cm}$ de large.

- qu'une même pluie peut avoir des effets extrêmement différents d'un site à l'autre à la même date (cf. par exemple la pluie du 5/01/86 à Epouville);

- que des pluies identiques peuvent avoir sur les mêmes sites des effets très différents selon le moment auquel elles interviennent (cf. pluies du 20/05/85 à Fongueusemare), qui correspond aussi à des conditions différentes d'état de surface des terrains;

- que certaines pluies très intenses n'ont pas d'effet érosif (20/05/85 à Fongueusemare), alors que d'autres. de très faible intensité, (28/04/85 à Epouville) en ont sur certains sites; là encore, les conditions d'état de surface ne sont pas identiques.

Les manifestations les plus graves semblent liées plutôt à de fortes hauteurs totales qu'à de fortes intensités.

\section{Influence des caractéristiques d'état de surface du terrain}

En tenant compte de l'ensemble des observations réalisées sur les bassins versants suivis, y compris si elles se rapportent non plus à une pluie bien délimitée mais à un groupe de pluies, nous disposons de 134 cas observés. Les notations d'état de surface de l'impluvium effectuées juste avant les pluies ou groupes de pluies correspondants permettent de situer ces observations dans un tableau qui fait intervenir: en colonnes, les critères qui contribuent à définir l'infiltrabilité: stade de formation des croûtes et présence ou absence d'empreintes de roues superficielles ;

- en lignes, les critères relatifs à la détention superficielle et à la circulation de l'excès d'eau : appréciation de la rugosité, présence ou absence d'un réseau de collecte prédéterminé.

Chaque observation ne peut être correctement resituée dans le tableau ainsi constitué que si on a bien identifié les zones qui jouent le rôle d'impluvium et celles où s'effectuent la collecte et la concentration du ruissellement. Ceci illustre bien l'importance, précédemment soulignée, du diagnostic sur les possibilités de communication entre les parcelles amont et aval, lorsque le bassin versant étudié se compose de plusieurs parcelles.

Chaque cas observé est noté en présence-absence selon qu'une aggravation de l'érosion a pu ou non être détectée par rapport à la situation précédente. Cette détection est évidemment plus facile lors des toutes premières étapes de formation des rigoles.

On obtient finalement le tableau 8. Celui-ci fait apparaître une ségrégation très marquée des valeurs des fréquences des cas d'érosion. On peut distinguer 3 domaines bien tranchés:

\section{Absence de phénomènes d'érosion}

Il s'agit de toutes les situations pour lesquelles l'im- 
TABLEAU 8

Fréquence des cas d'érosion en fonction de l'état de surface de l'impluvium (nombre de c'as avec déclenchement ou aggravation de l'érosion par rappor au nombre total de cas observés pour chaque type d'état de surface)

Frequency of erosion cases in relation to the catchment surface state (number of cases with erosion/total number of observed cases for a given type of surface state).

Stade de formation des croûtes de battance

F3

Fo

Fl

Transition

Fl-F2

F2

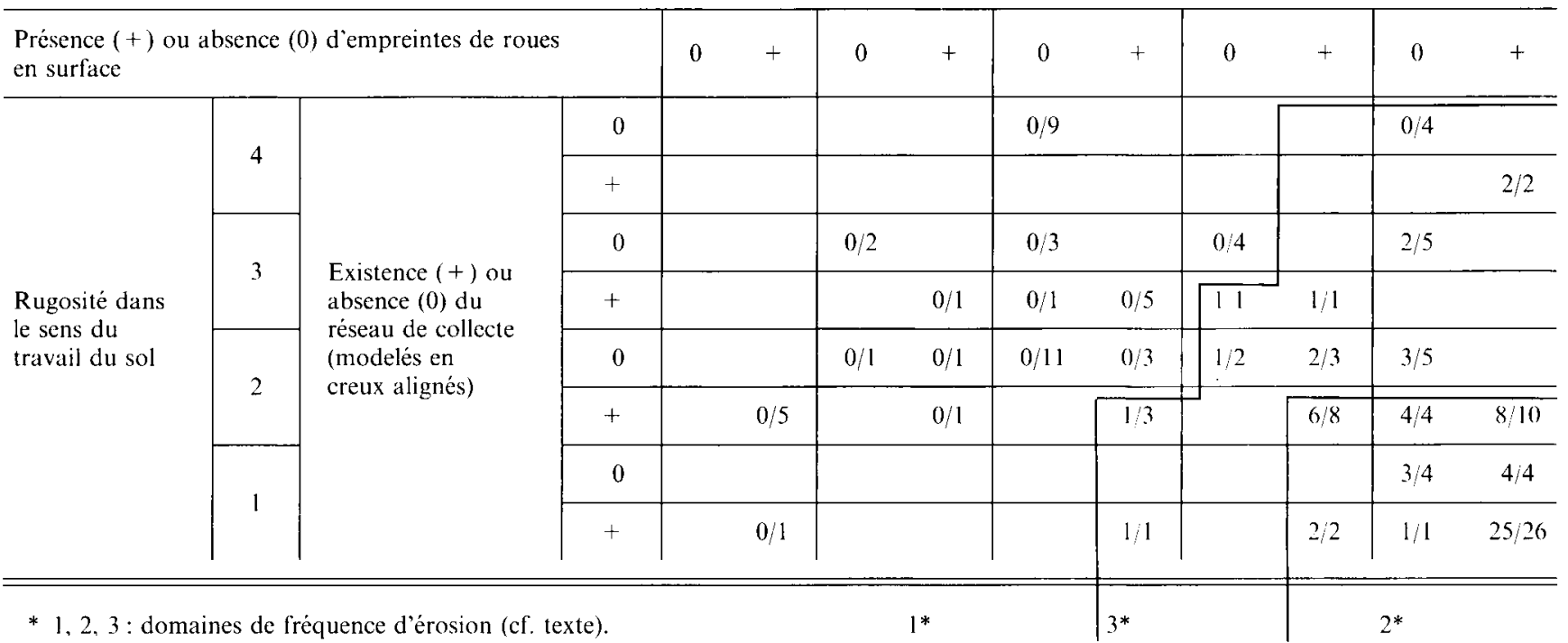

pluvium présente un faciès $F 3, F 0$ ou $F 1$, et des situations à faciès de transition ou F2 net qui, en l'absence de traces de roues, présentent par ailleurs une rugosité moyenne à forte. L'absence d'érosion semble pouvoir être imputée au fait d'une absence de ruissellement, soit en raison d'un excès d'eau faible ou nul dû à une infiltrabilité élevée, soit en raison d'une forte détention superficielle.

\section{Erosion presque systématique}

Il s'agit de situations où pratiquement tous les caractères défavorables sont réunis:

- impluvium dégradé : croûtes de battance de type sédimentaire ou faciès de transition avec traces de roues ;

- fortes possibilités de circulation et de concentration du ruissellement: rugosité très faible, modelé propice à la collecte.

3. Entre ces 2 zones du tableau s'interpose un domaine à érosion incertaine: il s'agit des situations à faciès F2, ou de transition, dont le modelé favorise peu la circulation de l'eau sans l'interdire totalement comme dans le premier cas. On y trouve également 2 cas à faciès $F 1$ mais faible rugosité et traces de roues.

Ce tableau est redevable d'une lecture à la fois diachronique et synchronique :

- pour un site donné, les caractères qui définissent la place d'une observation dans le tableau évoluent dans le temps: par exemple, la battance s'accompagne d'un déplacement vers la droite. La signification concrète des résultats obtenus est que l'apparition des rigoles est systématiquement consécutive à celle des croûtes sédimentaires avec un décalage d'autant plus bref que le réseau de collecte est plus efficace, ce qui est le cas des surfaces avec empreintes de roues ou de modelés billonnés dont les dépressions sont linéaires et peu rugueuses ;

pour une pluie donnée, l'existence ou l'absence d'un effet érosif sur chaque parcelle peuvent être aisément prévues à partir d'une typologie relativement simple des états de surface. Ainsi, à l'automne, une grande partic des figures d'érosion observées sont imputables aux chantiers d'arrachage des betteraves ou pommes de terre qui ne sont pas labourés immédiatement et émettent du ruissellement vers une parcelle située en contrebas. Celle-ci peut alors subir des dégâts sévères, sans contribuer elle-même au ruissellement. De la même façon, à l'automne comme au printemps, tous les semis présentant soit des croûtes sédimentaires développées (F2), soit un début de faciès sédimentaire avec traces de roues en surface sont susceptibles d'émettre du ruissellement même pour de faibles intensités pluviales. Inversement, les semis encore peu dégradés (parce que récemment réalisés), et les parcelles à modelé chaotique même assez dégradé (labours, déchaumage grossiers), présentent très peu de risques de circulation d'eau superficielle. donc d'érosion en aval.

Linterprétation et la discussion du tableau 8 se réfèrent donc essentiellement aux risques de ruissellement. Pour une évaluation plus complète des risques d'érosion, il faut faire intervenir la résistance du terrain au niveau des collecteurs. Les résultats disponibles sont 
en nombre insuffisant pour nous permettre de mettre en évidence de manière certaine l'influence de ce facteur : cependant, il semble bien que ce sont dans les situations à collecteur ameubli qu'on obtient les dégâts les plus spectaculaires. Ceci est corroboré par l'observation directe des rigoles de talweg des sites 6 et 7 , au niveau du franchissement de la limite entre les parcelles amont compactes (chantiers de récolte) et les parcelles aval meubles (semis de blé). Ce franchissement s'accompagne d'un élargissement et d'un approfondissement brutal de la rigole alors que les versants adjacents semés en blé n'apportent pas de ruissellement.

Ce rôle très probable de la compacité du terrain montre qu'il y a une certaine antinomie entre les critères de jugement de l'état structural appliqués à l'impluvium et au collecteur principal, ce qui renforce la nécessité de bien distinguer ces 2 zones.

Les chiffres de fréquence des cas d'érosion observés (tabl. 8) masquent en fait une forte variabilité de gravité des symptômes. Ainsi, sur les 30 cas du domaine à háut risque (faciès $F 2$, rugosité nulle), les notations de gravité vont de 0 à 3 avec une répartition très dispersée sur les classes intermédiaires. Cette dispersion ne doit pas surprendre : il aurait été aberrant que la variabilité des caractères influant sur le débit unitaire des écoulements (intensité des pluies, taille des impluviums, géométrie et topographie des collecteurs), ne se répercute pas sur les résultats obtenus. Par exemple, le cas de non-érosion observé dans le domaine à haut risque correspond à la pluie la moins importante (15/11/85 à Epouville) de tout l'échantillon de pluies observé. Inversement, les 2 cas d'érosion concomitants avec un faciès F1 correspondent à des pluies particulièrement intenses et abondantes.

\section{CONCLUSION}

D'un point de vue qualitatif (déclenchement ou non de l'érosion), il apparaît une excellente correspondarce entre 'les risques d'érosion et l'état de surface des parcelles, tel qu'il peut être appréhendé par observation rapide sur le terrain. Cette correspondance se dégage à partir du moment où l'on met en relation, de façon adéquate, l'observation du phénomène érosif avec l'impluvium qui lui est associé, et qui n'appartient pas nécessairement à la même parcelle.

Ce résultat a pu être établi dans des conditions de pluies et de topographie très variables, sans toutefois qu'aient pu être observés des événements pluvieux exceptionnellement agressifs. Les conclusions à en tirer concernent donc plutôt l'érosion du type chronique que celle de type catastrophique liée aux orages d'été. On remarquera d'ailleurs que l'analyse qui a été menée conduit à ne pas imputer exclusivement aux pluies le caractère catastrophique : celui-ci dépend également de la taille et de l'état de surface de l'impluvium qui reçoit la pluie.

Dans le contexte étudié, les risques d'érosion dépendent de façon très étroite de la formation des croûtes de battance et en second lieu du compactage superficiel. Dans des régions à sols moins instables que les limons du Pays de Caux, la hiérarchie de ces 2 processus serait peut-être inversée et il faudrait sans doute appréhender de façon plus précise les caractéristiques des empreintes de roues.

Il est clair d'ailleurs que l'analyse et la prévision des débits de ruissellement et des pertes en terre demanderont une caractérisation quantitative des paramètres hydrauliques (infiltrabilité, détention superficielle, délais de concentration, débits et flux, ...) et structuraux (porosité, rugosité, modelé de surface, ...) qui ont été invoqués pour expliquer les observations d'érosion. Mais, dès à présent, les résultats obtenus nous autorisent à discuter de l'effet des systèmes de culture en fonction de leur capacité à conférer aux terrains les caractéristiques qui se sont révélées pertinentes pour expliquer les phénomènes observés. Cette discussion fera l'objet d'un deuxième article.

Recule 10 mars 1988. Accepté le 20 juin 1988.

\section{REMERCIEMENTS}

Cette étude a été réalisée grâce au concours financicr du Ministèro de l'Agriculture (D.I.A.M.E.) et du Service d'Economie Régionale et de Développement Agricole de Haute-Normandie.

\section{RÉFÉRENCES BIBLIOGRAPHIQUES}

Auzet V., 1987. L'érosion des sols par l'eau dans les régions de grande culture: aspects agronomiques. Ministère de l'Environnement et Ministère de l'Agriculture, CEREG, Organisation-Environnement, 53 pages + annexes.

Boiffin J., 1984. La dégradation structurale des couches superficielles du sol sous l'action des pluies. Thèse D.I. INA-PG, $320 \mathrm{p}$. + annexes.

Boiffin J., 1986. Stages and time-dependency of soil crusting in situ, 91-98. In F. Callebaut, D. Gabriels, M. de Boodt. Assessment of soil surface sealing and crusting. Flanders Research Center for Soil Erosion and Soil conservation, Ghent, $374 \mathrm{p}$.

Boiffin J., Bresson L. M., 1987. Dynamique de formation des croûtes superficielles: Apport de l'analyse microscopique, 393-399. In N. Fedoroff, L. M. Bresson, M. A. Courty. Micromorphologie des sols-Soil Micromorphology. A.F.E.S., Plaisir, France, 686 p.

Boiffin J., Monnier M., 1986. Infiltration rate as affected by soil surface crusting caused by rainfall, 210-217. In F. Callebaut, G. Gabriels, M. de Boodt. Assessment of soil surface sealing and crusting. Flanders Reasearch Center for Soil Erosion and Soil Conservation, Ghent, $374 \mathrm{p}$
Boiffin J., Papy F., Peyre Y., 1986. Systèmes de production, systèmes de culture et risques d'érosion dans le Pavs de Caux. Doc. Multig. INAPG, INRA, Ministère de l’Agric. (DIAME) ; 154 p. + annexes. Boiffin J., Sebillotte M., 1976. Climat, stabilité structurale et battance. Essai d'analyse d'un comportement du sol au champ. Ann. Agron. 27 (3), 295-325.

Cogo N. P., Moldenhauer W. C., Foster G. R., 1984. Soil loss reductions from conservation tillage practices. Soil Sci. Soc. Am. J., 48 (2), 368-373.

Dexter A. R., 1977. Effect of rainfall on the surface micro-relief of tilled soil. J. Terramechanics, 14 (1), 11-22.

Ellison W. D., 1954. Mechanics of water erosion. C.R. $V^{\text {e }}$ Congrès Intern. Sc. Sol., 3, 380-387.

Foster G. R., Lane L. J., Nowlin J. D., Laflen J. M., Young R. A., 1981. Estimating erosion and sediment yield on field-size areas. Trans ASAE, 24 (5), 1253-1262.

Foster G. R., Lane L. J., Mildner W. F., 1985. Seasonally ephemeral cropland gully erosion. Proceedings of the ARS-SCS. Natural resources modelling workshop, Washington, D.C., USDA-Agricultural Research Service. 
Fullen M., 1985. Compaction, hydrological processes and soil erosion on loamy sands in East Shropshire, England. Soil Tillage Res., 6, 17-29.

Govers G., 1985. Selectivity and transport capacity of thin flows in relation to rill erosion. Catena, 12, 35-49.

Hudson N. W., 1981. Soil conservation. 2nd Ed. Batsford Academic and Educational Ltd. London, $324 \mathrm{p}$.

Lyle W. M., Smerdon E. T., 1965. Relation of compaction and other properties to erosion resistance of soils. Trans ASAE, 8 (3), 419-422.

Mitchell J. K., Jones A. B., 1978. Micro-relief surface depression storage changes during rainfall events and their application to rainfall runoff models. Water Resources Bulletin, 14 (4), 777-802.

Monnier G., Boiffin J., 1986.Effect of the agricultural use of soils on water erosion: the case of cropping systems in Western Europe, 17-32. In G. Chisci, R. P. C. Morgan. Soil erosion in the European Community. Impact of Changing Agriculture. Balkema, Rotterdam, Boston, $233 \mathrm{p}$

Monnier G., Boiffin J., Papy F., 1986. Réflexions sur l'érosion hydrique en conditions climatiques et topographiques modérées: Cas des systèmes de grande culture de l'Europe de l'Ouest. Cah. ORSTOM, sér. Pédol., 12 (2), 123-131.

Monteith N. H., 1974. The role of surface roughness in runoff. Soil conservation. J. of New South Wales, 30 (1), 42-45.

Moore I. D., Larson C. L., 1979. Estimating micro-relief surface storage from point data. Trans $A S A E, 5$ (22), 1073-1077.
Onstad C. A., 1984. Depressional storage on tilled soil surfaces. Trans ASAE, 27 (3), 729-732.

Rauws G., 1987. The initiation of rills on plane beds of non-cohesive sediments. Catena Suppl., 8, 107-108.

Savat J., 1979. Laboratory experiments on erosion and deposition of loess by laminar sheet flow and turbulent flow, 139-144. In H. Vogt, T. Vogt. Colloque sur l'érosion agricole des sols en milieu tempéré non méditerranéen. Université Louis Pasteur, Strasbourg, 251 p.

Savat J., De Ploey J., 1982. Sheetwash and rill development by surface flow, 113-126. In R. Bryan, A. Yair. Badland Geomorphology and Piping. Geo Books, Cambridge, 218 p.

Steichen J. M., 1984. Infiltration and random roughness of a tilled sand untilled claypan soil. Soil and Tillage Res., 4, 251-262.

Spomer R. G., Hjelmfelt A. T., 1986. Concentrated flow erosion on conventional and conservation tilled watersheds. Trans ASAE, 29 (1), 124-127.

Voorhees W. B., Young R. A., Lyles L., 1979. Wheel traffic considerations in erosion research. Transactions of the ASAE, 4 (22), 786-790.

Wischmeier W. H., Smith D. D., 1965. Predicting rainfall erosion losses from cropland East of Rocky Mountains. Agric. Handbook 282, USDA.

Young R. A., Wiersma J. L., 1973. The role of rainfall impact in soil detachment and transport. Water Resources Res., 9 (6), 1629-1636.

Zobeck T. M., Onstad C. A., 1987. Tillage and rainfall effects on random roughness : a review. Soil and Tillage Res., 9, 1-20. 